\title{
Application of Power Geometry and Normal Form Methods to the Study of Nonlinear ODEs
}

\author{
Victor Edneral ${ }^{1,2, \star}$ \\ ${ }^{1}$ Skobeltsyn Institute of Nuclear Physics, Lomonosov Moscow State University, Leninskie Gory 1 (2), \\ Moscow, 119991, Russian Federation \\ ${ }^{2}$ Peoples's Friendship University of Russia (RUDN University), Miklukho-Maklaya street 6, \\ Moscow, 117198, Russian Federation
}

\begin{abstract}
This paper describes power transformations of degenerate autonomous polynomial systems of ordinary differential equations which reduce such systems to a nondegenerative form. Example of creating exact first integrals of motion of some planar degenerate system in a closed form is given.
\end{abstract}

\section{Introduction}

We consider an autonomous system of ordinary differential equations of the form

$$
\frac{d x_{i}}{d t} \stackrel{\text { def }}{=} \dot{x}_{i}=\varphi_{i}(X, P), \quad i=1,2,
$$

where $X=\left(x_{1}, x_{2}\right) \in \mathbb{C}^{2}$ and $\varphi_{i}(X, P)$ are polynomials in the variables $X$ and involve the parameters $P=\left(b, a_{0}, a_{1}, b_{0}, b_{1}\right)$.

A method of analysis of the integrability of the system (1) based on Power Transformations [1] and computation of normal forms near stationary solutions of transformed systems (see [2] and Ch. II in [3]) was proposed in $[4,5]$.

In a neighborhood of the stationary point $X=0$, the system (1) can be written in the form

$$
\dot{X}=\hat{A} X+\tilde{\Phi}(X, P),
$$

where $\tilde{\Phi}(X, P)$ has no terms linear in $X$.

Let $\lambda_{1}$ and $\lambda_{2}$ be eigenvalues of the matrix $\hat{A}$. If at least one is different from zero, then the stationary point $X=0$ is called elementary stationary point. In this case, the system (2) has a normal form (see, e.g. Ch. II in [3]). If both eigenvalues vanish, then the stationary point $X=0$ is called nonelementary stationary point. In this case, there is no normal form for the system (2). But using power transformations, we can split the nonelementary stationary point $X=0$ to a set of elementary stationary points [1]. For each of these elementary stationary points, we can compute the normal form and obtain the conditions of local integrability.

\footnotetext{
^e-mail: edneral_vf@rudn.university
} 
In the present paper, we demonstrate how this approach can be applied to the study of local and global integrability in the case of a planar system near the stationary point $X^{0}=0$ of high degeneracy in the case of the system

$$
\begin{aligned}
& \dot{x}=\alpha y^{3}+\beta x^{3} y+\left(a_{0} x^{5}+a_{1} x^{2} y^{2}\right), \\
& \dot{y}=\gamma x^{2} y^{2}+\delta x^{5}+\left(b_{0} x^{4} y+b_{1} x y^{3}\right) .
\end{aligned}
$$

This system is a subfamily of the system introduced in [6].

Systems with a nilpotent matrix of the linear part were thoroughly studied by Lyapunov and others. In the system (3), there is no linear part, and the first approximation is not homogeneous. This is the simplest case of a planar system without linear part and with Newton's open polygon (see Ch. II in [3]) consisting of a single edge. In the general case, such problems have not been studied yet. However, a particular case of the system of this type was considered in [6], where the authors put $-\alpha=\delta=1$ and $3 \beta+2 \gamma=0$. Further, the authors of [6] studied the Hamiltonian subcase of this system under the additional assumption that the Hamiltonian function is expandable into the product of only square-free factors.

We study the following problem: under what conditions imposed on the parameters, the system (3) is locally or globally integrable when the system is non-Hamiltonian.

We discuss the first quasi homogeneous approximation of the system (3) and the necessary conditions of local integrability. Then we calculate the set of such conditions for the complete system (3). After that, we prove that these conditions are sufficient for the global integrability by computing corresponding the first integrals of motion. First, we do it for the case when $b^{2} \neq 2 / 3$ in the system (6), and after that for a subcase of the case $b^{2}=2 / 3$.

\section{Problem Formulation}

We start by investigating the case when the first quasi homogeneous approximation of (3) considered in $[5,7]$ has the form

$$
\dot{\tilde{x}}=\alpha \tilde{y}^{3}+\beta \tilde{x}^{3} \tilde{y}, \quad \dot{\tilde{y}}=\gamma \tilde{x}^{2} \tilde{y}^{2}+\delta \tilde{x}^{5},
$$

where $\alpha \neq 0$ and $\delta \neq 0$. Using the linear transformation $x=\sigma \tilde{x}, y=\tau \tilde{y}$ we can fix two nonzero parameters in (4) and obtain the system

$$
\dot{x}=-y^{3}-b x^{3} y, \quad \dot{y}=c x^{2} y^{2}+x^{5} .
$$

Each autonomous planar quasi homogeneous system (4) has an integral, but this integral can be non analytic. We have are interested in the analytic integrability of (3), so we look for the conditions on the parameters under which the system (5) is locally or globally analytically integrable.

The following result was proved in $[5,7]$ :

Theorem 1. In the case $D \stackrel{\text { def }}{=}(3 b+2 c)^{2}-24 \neq 0$, the system (5) is locally integrable if and only if the number $N=(3 b-2 c) / \sqrt{D}$ is rational. The system (5) is also locally integrable in the case $b^{2}=2 / 3$.

In this paper, at first we study the simple particular case $c=1 / b$, then $N=1$ and $D=(3 b-2 / b)^{2} \neq$ 0 . Due to the Theorem 1, the first quasi homogeneous approximation has an analytic integral but it is not a Hamiltonian system. Then we study the case $b^{2}=2 / 3, D=0$.

We study the integrability problem for the entire system (3) with the first quasi homogeneous approximation (5) representing the system in the form

$$
\begin{aligned}
& \frac{d x}{d t}=-y^{3}-b x^{3} y+a_{0} x^{5}+a_{1} x^{2} y^{2}, \\
& \frac{d y}{d t}=\frac{1}{b} x^{2} y^{2}+x^{5}+b_{0} x^{4} y+b_{1} x y^{3} .
\end{aligned}
$$


Thus, we consider the system with five arbitrary parameters $a_{i}, b_{i},(i=0,1)$ and $b \neq 0$.

\section{Power Transformation}

First, we rewrite (6) in a non-degenerate form. This can be done by using the Blow-Up process [9]. We can use here the Power Transformation (see Ch. I, §1.8 in [3])

$$
x=u v^{2}, \quad y=u v^{3} .
$$

The power transformation

$$
\begin{aligned}
& y_{1}=x_{1}^{\alpha_{1,1}} x_{2}^{\alpha_{1,2},}, \\
& y_{2}=x_{1}^{\alpha_{2,1}} x_{2}^{\alpha_{2,2},}
\end{aligned}
$$

has the inverse one

$$
\begin{aligned}
& x_{1}=y_{1}^{\beta_{1,1}} y_{2}^{\beta_{1,2}}, \\
& x_{2}=y_{1}^{\beta_{2,1}} y_{2}^{\beta_{2,2}},
\end{aligned}
$$

where $\hat{\beta}=\hat{\alpha}^{-1}$. In fact, the above transformations are linear with respect to the logarithms of the variables

$$
\begin{array}{ll}
\log y_{1}=\alpha_{1,1} \log x_{1}+\alpha_{1,2} \log x_{2}, & \log x_{1}=\beta_{1,1} \log y_{1}+\beta_{1,2} \log y_{2}, \\
\log y_{2}=\alpha_{2,1} \log x_{1}+\alpha_{2,2} \log x_{2}, & \log x_{2}=\beta_{2,1} \log y_{1}+\beta_{2,2} \log y_{2}
\end{array}
$$

The equation (6) can be rewritten as

$$
\begin{aligned}
& \frac{d \log x}{d t}=-x^{-1} y^{3}-b x^{2} y+a_{0} x^{4}+a_{1} x y^{2}, \\
& \frac{d \log y}{d t}=\frac{1}{b} x^{2} y+x^{5} y^{-1}+b_{0} x^{4}+b_{1} x y^{2} .
\end{aligned}
$$

In the two-dimension space of degrees of the variables $x$ and $y$ the monomials of equations (8) form two parallel lines. The task now is to perform a rotation of the lines clockwise or counterclockwise till they become parallel to the abscissa or ordinate axes. The parallel to these lines vector before and after the needed transformation is

$$
\left(\begin{array}{ll}
1 & 1 \\
2 & 3
\end{array}\right)\left(\begin{array}{c}
3 \\
-2
\end{array}\right)=\left(\begin{array}{l}
1 \\
0
\end{array}\right)
$$

is parallel to the abscissa line.

The transformation (7) corresponds to the transposed matrix above

$$
\left(\begin{array}{l}
\log x \\
\log y
\end{array}\right)=\left(\begin{array}{ll}
1 & 2 \\
1 & 3
\end{array}\right)\left(\begin{array}{l}
\log u \\
\log v
\end{array}\right)
$$

With the time rescaling $u^{2} v^{7} d t=d \tau$, the system (6) is rewritten in the form

$$
\begin{aligned}
& \frac{d u}{d \tau}=-3 u-[3 b+(2 / b)] u^{2}-2 u^{3}+\left(3 a_{1}-2 b_{1}\right) u^{2} v+\left(3 a_{0}-2 b_{0}\right) u^{3} v, \\
& \frac{d v}{d \tau}=v+\left[b+\frac{1}{b}\right] u v+u^{2} v+\left(b_{1}-a_{1}\right) u v^{2}+\left(b_{0}-a_{0}\right) u^{2} v^{2} .
\end{aligned}
$$

The transformation of variables (7) is not unique. We are searching for a substitution such that: 
- the ration of the eigenvalues of the linear part $\lambda_{1} / \lambda_{2}$ would be a negative rational number;

- the value of the resonance of the linear part of the resulting equation is expected to be minimal;

- the inverse transformation has integer degrees of the variables. So the wanted matrix is unimodular.

Under the power transformation (7), the point $x=y=0$ blows up into two straight invariant lines $u=0$ and $v=0$. Along the line $u=0$, system (9) has the stationary point $u=v=0$. Along the second line $v=0$, this system has four elementary stationary points

$$
u=0, \quad u=-\frac{1}{b}, \quad u=-\frac{3 b}{2}, \quad u=\infty .
$$

The necessary condition for local integrability of the system (6) at the point $x=y=0$ is the local integrability near each of the stationary point above.

Lemma 1. The system (9) is locally integrable near the points $u=v=0$ and $u=\infty, v=0$.

This lemma was proved in [5,7]. Thus, conditions of local integrability are to be found at the other two stationary points (10). Then we will get the conditions of local integrability of system the (6) near the original point $(0,0)$. For these purposes we use the condition $A$. Since it would take a lot of space, and because it was presented earlier in several papers $(\operatorname{see}[2,3])$ we do not repeat it here. The condition $A$ was formulated and applied to the considered problem in [4]. For two-dimensional systems, it is sufficient condition of their local integrability. It is an algebraic condition on the coefficients of the normal form. In our case, it can be presented as a system of algebraic equations. We have computed the condition $A$ with the program described in [8].

Let us consider the stationary point $u=-1 / b, v=0$. There are two solutions for the corresponding subset of equations at $b \neq 0$ [7]

$$
a_{0}=0, \quad a_{1}=-b_{0} b, \quad b_{1}=0, \quad b^{2} \neq 2 / 3
$$

and

$$
a_{0}=a_{1} b, \quad b_{0}=b_{1} b, \quad b^{2} \neq 2 / 3 .
$$

The consideration of the stationary point $u=-3 b / 2, v=0$ under the condition (12) gives three additional two-parameter solutions (depending on $a_{1}$ and $b$ ), see [7]

$$
\begin{aligned}
& \text { 1) } b_{1}=-2 a_{1}, \quad a_{0}=a_{1} b, \quad b_{0}=b_{1} b, \quad b^{2} \neq 2 / 3 \text {, } \\
& \text { 2) } b_{1}=(3 / 2) a_{1}, \quad a_{0}=a_{1} b, \quad b_{0}=b_{1} b, \quad b^{2} \neq 2 / 3 \text {, } \\
& \text { 3) } b_{1}=(8 / 3) a_{1}, \quad a_{0}=a_{1} b, \quad b_{0}=b_{1} b, \quad b^{2} \neq 2 / 3 \text {. }
\end{aligned}
$$

Thus, we proved $([5,7])$

Theorem 2. The conditions (11) and (13) form the set of necessary conditions of local integrability of the system (9) near all its stationary points and the local integrability of the system (6) at the stationary point $x=y=0$.

\section{First Integrals of Motion}

The conditions presented in Theorem 2 as necessary conditions for the local integrability of system (6) can be considered as good candidates for sufficient conditions of global integrability. However, it is necessary to prove the sufficiency of these conditions by independent methods. It is necessary to do 
it for each of four conditions (11) and (13) at every stationary point $u=-3 b / 2, v=0$ and $u=-1 / b$, $v=0$, for $b^{2} \neq 2 / 3$.

In [10], we have calculated first integrals of system (9) for all cases (11), (13) (mainly by the Darboux method, see, e.g., [11]).

We have found four families of solutions which covered all the cases mentioned above:

1. At $a_{0}=0, a_{1}=-b_{0} b, \quad b_{1}=0$ :

$$
I_{1 u v}=u^{2}(3 b+2 u) v^{6}, \quad I_{1 x y}=2 x^{3}+3 b y^{2} .
$$

2. At $b_{1}=-2 a_{1}, a_{0}=a_{1} b, \quad b_{0}=b_{1} b$ :

$$
I_{2 u v}=u^{2} v^{6}\left(3 b+u\left(2-6 a_{1} b v\right)\right), \quad I_{2 x y}=2 x^{3}-6 a_{1} b x^{2} y+3 b y^{2} .
$$

3. At $b_{1}=3 a_{1} / 2, \quad a_{0}=a_{1} b, \quad b_{0}=b_{1} b$ :

$$
\begin{aligned}
I_{3 u v} & =\frac{4-4 a_{1} u v+3^{5 / 6} a_{1} \times{ }_{2} F_{1}(2 / 3,1 / 6 ; 5 / 3 ;-2 u /(3 b)) \times u v(3+2 u / b)^{1 / 6}}{u^{1 / 3} v(3 b+2 u)^{1 / 6}}, \\
I_{3 x y} & =\frac{a_{1} x^{2}\left(-4+3^{5 / 6}{ }_{2} F_{1}\left(2 / 3,1 / 6 ; 5 / 3 ;-2 x^{3} /\left(3 b y^{2}\right)\right) \times\left(3+2 x^{3} /\left(b y^{2}\right)\right)^{1 / 6}\right)+4 y}{y^{4 / 3}\left(3 b+2 x^{3} / y^{2}\right)^{1 / 6}},
\end{aligned}
$$

4. At $b_{1}=8 a_{1} / 3, a_{0}=a_{1} b, \quad b_{0}=b_{1} b$ :

$$
I_{4 u, v}=\frac{u\left(3+2 a_{1}^{2} b u\right)+6 a_{1} b v}{3 u\left[u^{3}\left(6+a_{1}^{2} b u\right)+6 a_{1}^{2} b u^{2} v+9 b v^{2}\right]^{1 / 6}}-8 a_{1} \sqrt{-b} / 3^{5 / 3} B_{6+a_{1}} \sqrt{-6 b u}+3 v \sqrt{-6 b / u^{3}}(5 / 6,5 / 6),
$$

where $B_{t}(a, b)$ is the incomplete beta function and ${ }_{2} F_{1}(a, b ; c ; z)$ is the hypergeometric function [12].

The first integrals and the solutions do not have any singularities at the values $b^{2}=2 / 3$, but the approach by which these solutions were found, has the limitation $b^{2} \neq 2 / 3$, so there could exist possible additional integrals for these values. Thus, we need to study the case $b^{2}=2 / 3$ separately.

\section{Case $b^{2}=2 / 3$, subcase $3 a_{0}-2 b_{0}=b\left(3 a_{1}-2 b_{1}\right)$}

Let us consider the case $b=\sqrt{2 / 3}$ (the case $b=-\sqrt{2 / 3}$ gives the same results). For this value of $b$, both stationary points $u=-3 b / 2, v=0$ and $u=-1 / b, v=0$ are collapsing, and after the shift $u \rightarrow w-1 / b$, we get the degenerate system instead of (9)

$$
\begin{aligned}
\frac{d w}{d \tau}= & -\frac{3 v}{2 b}\left[\left(3 a_{0}-2 b_{0}\right)-b\left(3 a_{1}-2 b_{1}\right)\right]+w v\left(\frac{27}{2 a_{0}-3 \sqrt{6}} a_{1}-9 b_{0}+2 \sqrt{6} b_{1}\right) \\
& +\sqrt{6} w^{2}+w^{2} v\left(-9 \sqrt{\frac{3}{2}} a_{0}+3 a_{1}+3 \sqrt{6} b_{0}-2 b_{1}\right)-2 w^{3}+w^{3} v\left(3 a_{0}-2 b_{0}\right), \\
\frac{d v}{d \tau}= & -\frac{\sqrt{6}}{6} w v+v^{2}\left(-\frac{3}{2} a_{0}+\sqrt{\frac{3}{2}} a_{1}+\frac{3}{2} b_{0}-\sqrt{\frac{3}{2}} b_{1}\right) \\
& +w^{2} v+w v^{2}\left(\sqrt{6} a_{0}-a_{1}-\sqrt{6} b_{0}+b_{1}\right)+w^{2} v^{2}\left(-a_{0}+b_{0}\right) .
\end{aligned}
$$

At the stationary point $w=v=0$ this system has zero eigenvalues, so we need to apply the power transformation once again. In [7], we applied the transformation

$$
v \rightarrow r^{2} w, \quad \dot{v} \rightarrow 2 \dot{r} r w+r^{2} \dot{w},
$$


and obtained systems with resonances of 19th and 27th orders. We have calculated the corresponding normal form with 4 free parameters till 19th order, but to find new solutions we need to compute the 27 th order resonance. This resonance exists only if $3 a_{0}-2 b_{0} \neq b\left(3 a_{1}-2 b_{1}\right), b^{2}=2 / 3$, and its calculation is very complicated. We postpone the investigation of this subcase.

We see that in the systems (14), the coefficient of $v$ in the linear part of the first equation is equal to zero if $3 a_{0}-2 b_{0}=b\left(3 a_{1}-2 b_{1}\right)$. So we have the special subcase

$$
3 a_{0}-2 b_{0}=b\left(3 a_{1}-2 b_{1}\right), \quad b^{2}=2 / 3 .
$$

For this subcase, we use the transformation

$$
u \rightarrow w-1 / b, \quad v \rightarrow w r, \quad \dot{v} \rightarrow \dot{w} r+w \dot{r}, \quad \hat{\alpha}=\left(\begin{array}{ll}
1 & 0 \\
1 & 1
\end{array}\right),
$$

with time scaling by division of the equations by $w / \sqrt{6}$, so $\tilde{\tau}=w \tau / \sqrt{6}$. Then, from (9) we get

$$
\begin{aligned}
\frac{d w}{d \tilde{\tau}}= & 6 w+3\left(3 a_{1}-2 b_{1}\right) r w-2 \sqrt{6} w^{2}-2 \sqrt{6}\left(3 a_{1}-2 b_{1}\right) r w^{2}+2\left(3 a_{1}-2 b_{1}\right) r w^{3}, \\
\frac{d r}{d \tilde{\tau}}= & -7 r-\left(9 a_{1}-\sqrt{\frac{3}{2}} b_{0}-5 b_{1}\right) r^{2}+3 \sqrt{6} r w \\
& +\left(7 \sqrt{6} a_{1}-2 b_{0}-13 \sqrt{\frac{2}{3}} b_{1}\right) r^{2} w-\left(8 a_{1}-\sqrt{\frac{3}{2}} b_{0}-\frac{16}{3} b_{1}\right) r^{2} w^{2} .
\end{aligned}
$$

This is a three-parameter system with the resonance of the 13th order at the stationary point $w=0$, $r=0$ along the invariant line $w=0$. Along this line, there is also another stationary point. It is possible to prove the local integrability of the system at these points and they do not supply any additional restrictions on the parameters.

We have calculated the normal form for (17) till the 26th order and obtained two equations for the condition $A$. They are a $13=0$ and a $26=0$, where a 13 and a26 are given in [13]. Each of these equations is homogeneous in the parameters $a_{1}, b_{0}, b_{1}$ of the system (6) of the sixth and twelfth orders correspondingly. Both a13 and a26 are equal to zero for the solutions (11) and (13).

Homogeneous algebraic equations in three variables can be reduced to inhomogeneous equations in two variables. Let us assume that $a_{1} \neq 0$. In this case, we substitute $b_{0}=c_{0} a_{1}, b_{1}=c_{1} a_{1}$ and obtain the system of two equations in two variables a13 $\left(c_{0}, c_{1}\right)=0$, a26 $\left(c_{0}, c_{1}\right)=0$. The resultant of a13 $\left(c_{0}, c_{1}\right)$ and a26 $\left(c_{0}, c_{1}\right)$ in each of two variables $c_{0}$ and $c_{1}$ is identically equal to zero. So it is sufficient to solve only the equation $\mathrm{a} 13\left(c_{0}, c_{1}\right)=0$ and check these solutions by conditions of the higher order (i.e. by a26 $=0$, a39 $=0, \ldots$ ).

It is interesting that the condition $A$ of the 19 th order $(\mathrm{a} 19)$ from $[7,14]$ under the equality $3 a_{0}-$ $2 b_{0}=b\left(3 a_{1}-2 b_{1}\right)$ is identically equal to a13 up to the multiplication by a constant.

The a13 can be factorized as the product of four factors including $a_{1}^{6}$ :

$$
\begin{aligned}
\mathrm{a} 13= & 48\left(c_{1}-3 / 2\right) \times\left(c_{0}-1 / 12 \sqrt{6} c_{1}+1 / 2 \sqrt{6}\right)^{2} \\
& \times\left[409790784 c_{0}^{3}-104 \sqrt{6} c_{0}^{2}\left(-9152256+3385633 c_{1}\right)-\right. \\
& -208 c_{0}\left(-10917702+c_{1}\left(-360720+3319927 c_{1}\right)\right)+ \\
& \left.+\sqrt{6}\left(-718439040+c_{1}\left(2461047528+c_{1}\left(-1944898681+441207868 c_{1}\right)\right)\right)\right] \times a_{1}^{6} .
\end{aligned}
$$

From the first two factors, we get two two-parametric solutions $c_{1}=3 / 2$ and $c_{1}=6+2 \sqrt{6} c_{0}$ or

$$
\begin{array}{lll}
b_{1}=3 a_{1} / 2, & a_{0}=\left(2 b_{0}+b\left(3 a_{1}-2 b_{1}\right)\right) / 3, & b=\sqrt{2 / 3}, \\
b_{1}=6 a_{1}+2 \sqrt{6} b_{0}, & a_{0}=\left(2 b_{0}+b\left(3 a_{1}-2 b_{1}\right)\right) / 3, & b=\sqrt{2 / 3} .
\end{array}
$$


For the solutions (18), we calculate the normal form of (17) till the 36th order and obtain for each solution that it is an integrable diagonal linear system. The condition $a_{1}=0$ gives only one parametric solutions of the system a13 $=0, a 26=0$. The same result is obtained from the study of the cubic factor in a13 above. Therefore we do not consider that case here.

The corresponding first integrals of (17) are

$$
\begin{aligned}
I_{1 r w}= & w^{-7 / 6}\left(1-\sqrt{\frac{2}{3}} w\right)^{-1 / 3}\left[-9 a_{1}+3 \sqrt{6} b_{0}-\frac{42}{r}-6\left(\sqrt{6} a_{1}+5 b_{0}\right) w+2\left(9 a_{1}+4 \sqrt{6} b_{0}\right) w^{2}\right. \\
& \left.-2^{1 / 6}\left(9 \sqrt{2} a_{1}+8 \sqrt{3} b_{0}\right) w^{5 / 3}(-\sqrt{6}+2 w)^{1 / 3} \times{ }^{2} F_{1}(-1 / 2,1 / 3 ; 1 / 2 ; \sqrt{2 / 3} / w)\right], \\
I_{2 r w}= & r^{3-\frac{3 a_{1}}{3 a_{1}+\sqrt{6} b_{0}}} \cdot w^{\frac{7}{3}+\frac{7 b_{0}}{3 \sqrt{6} a_{1}+6 b_{0}}} \cdot(1-\sqrt{2 / 3} w)^{\frac{-a_{1}}{3 a_{1}+\sqrt{6} b_{0}}} \times\left\{\frac{-6+2 \sqrt{6} w}{6 a_{1}+3 \sqrt{6} b_{0}}+r[3+2 w(-\sqrt{6}+w)]\right\} .
\end{aligned}
$$

Back to the original variables $x, y$ of equation (6), these integrals up to the multiplication by a constant have the form:

$$
\begin{aligned}
I_{1 x y}= & \frac{y}{x^{2}}\left(\sqrt{6}+\frac{2 x^{3}}{y^{2}}\right)^{-7 / 6}\left(\frac{x^{3}}{y^{2}}\right)^{2 / 3} \times\left\{42 \sqrt{6}+\frac{1}{x y^{3}}\left[-36 a_{1} x^{6}-16 \sqrt{6} b_{0} x^{6}\right.\right. \\
& +84 x^{4} y-24 \sqrt{6} a_{1} x^{3} y^{2}-36 b_{0} x^{3} y^{2}+2^{1 / 3}\left(\frac{x^{3}}{y^{2}}\right)^{1 / 3} y^{2} \cdot\left(\sqrt{6}+\left(\frac{x^{3}}{y^{2}}\right)^{2 / 3}\right) \\
& \left.\left.\times\left(2\left(9 a_{1}+4 \sqrt{6} b_{0}\right) x^{3}+3\left(3 \sqrt{6} a_{1}+8 b_{0}\right) y^{2}\right) \times{ }_{2} F_{1}\left(-1 / 2,1 / 3 ; 1 / 2 ; \frac{3 y^{2}}{3 y^{2}+\sqrt{6} x^{3}}\right)\right]\right\}, \\
I_{2 x y}= & y \cdot\left(\sqrt{2 / 3}+\frac{x^{3}}{y^{2}}\right)^{-\frac{1}{2}+\frac{a_{1}}{-6 a_{1}-2 \sqrt{6} b_{0}}}\left(\frac{x^{2}}{y}\right)^{-\frac{a_{1}}{3 a_{1}+\sqrt{6} b_{0}}} \times\left\{3+\frac{x^{2}}{y^{2}}\left[\sqrt{6} x+3\left(2 a_{1}+\sqrt{6} b_{0}\right) y\right]\right\} .
\end{aligned}
$$

\section{Conclusions}

For the five-parameter non-Hamiltonian planar system (6), we have found six sets of two-parametric necessary conditions on parameters under which the system is locally integrable near the degenerate point $x=y=0$. These sets of conditions are also sufficient for the local and global integrability of the system (6). These six expressions for the first integrals of motion have been checked by direct substitution to the original system.

It is very interesting that the local study of the different stationary points of the system provides a step in proceeding from local to global analysis.

\section{Acknowledgements}

The publication was prepared with the support of the "RUDN University Program 5-100" and by the grant NSh-7989.2016.2 of the President of Russian Federation. The author is grateful to Professor Alexander D. Bruno from Keldysh Institute of Applied Mathematics of RAS for many fruitful ideas and to Professor Valery G. Romanovski from Maribor University (Slovenia) for significant contribution at works with Darboux factors.

\section{References}

[1] A.D. Bruno ed., Power Geometry in Algebraic and Differential Equations (Elsevier Science, Amsterdam, 2000) 396 pp. 
[2] A.D. Bruno, Trans. Moscow Math. Soc. 25, 131-288 (1971), 26, 199-239 (1972)

[3] A.D. Bruno, Local Methods in Nonlinear Differential Equations (Springer-Verlag, Berlin, 1989) X, $348 \mathrm{pp}$.

[4] A.D. Bruno and V.F. Edneral, Doklady Mathem. 79 (1), 48-52 (2009)

[5] A.D. Bruno and V.F. Edneral, Proceedings of CASC 2009 (LNCS 5743, Springer, Heidelberg, 2009) $45-53$

[6] A. Algaba A, E. Gamero, and C. Garcia, Nonlinearity 22, 395-420 (2009)

[7] A.D. Bruno and V.F. Edneral, Proceedings of CASC 2013 (LNCS 8136, Springer, Heidelberg, 2013) 75-87

[8] V.F. Edneral, Proceedings of CASC 2007 (LNCS 4770, Springer, Heidelberg, 2007) 134-142

[9] F. Dumortier, J. Llibre, J.C. Artés, Qualitative Theory of Planar Differential Systems (Springer, Berlin, Heidelberg, New York, 2006) XVI, 302 pp.

[10] V. Edneral and V.G. Romanovski, Proceedings of CASC 2010 (LNCS 6244, Springer, Heidelberg, 2010) 97-105

[11] V.G. Romanovski and D.S. Shafer, The Center and Cyclicity Problems: A Computational Algebra Approach (Birkhäuser, Boston, 2009) 330 pp.

[12] H. Bateman and A. Erdélyi, Higher Transcendental Functions, volume I (McGraw-Hill Book Comp., New York, Toronto, London, 1953) 316 pp.

[13] http://theory.sinp.msu.ru/ edneral/CASC2017/a13-26.txt

[14] http://theory.sinp.msu.ru/ edneral/CASC2017/a19.txt

[15] C. Christopher, P. Mardešić, and C. Rousseau, J. Dyn. Control Sys. 9, 311-363 (2003) 